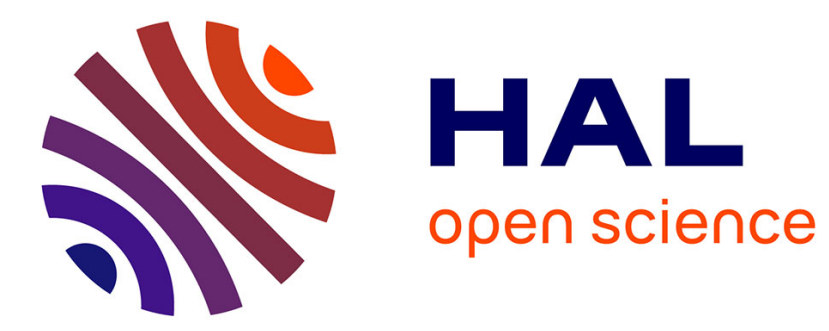

\title{
Mesoscopic Tube Model of Fluids Composed of Wormlike Micelles
}

Miroslav Grmela, Francisco Chinesta, Amine Ammar

\section{To cite this version:}

Miroslav Grmela, Francisco Chinesta, Amine Ammar. Mesoscopic Tube Model of Fluids Composed of Wormlike Micelles. Rheologica Acta, 2010, 49 (5), pp.495-506. 10.1007/s00397-009-0428-y . hal01007027

\section{HAL Id: hal-01007027 \\ https://hal.science/hal-01007027}

Submitted on 30 Apr 2017

HAL is a multi-disciplinary open access archive for the deposit and dissemination of scientific research documents, whether they are published or not. The documents may come from teaching and research institutions in France or abroad, or from public or private research centers.
L'archive ouverte pluridisciplinaire HAL, est destinée au dépôt et à la diffusion de documents scientifiques de niveau recherche, publiés ou non, émanant des établissements d'enseignement et de recherche français ou étrangers, des laboratoires publics ou privés. 


\title{
Mesoscopic Tube Model of Fluids Composed of Wormlike Micelles
}

\author{
Miroslav Grmela ${ }^{1 *}$ Francisco Chinesta ${ }^{2}$, Amine Ammar $^{3}$
}

${ }^{1}$ Ecole Polytechnique de Montreal, C.P.6079 suc. Centre-ville, Montreal, H3C 3A7, Quebec, Canada

2 EADS Corporate International Chair GeM, Ecole Centrale de Nantes BP 92101, 44321 Nantes, cedex 3, France

${ }^{3}$ Laboratoire de Rheologie, INPG, UJF, CNRS (UMR 5520), 1301 rue de la piscine, BP 53 Domaine Universitaire, F-38041 Grenoble Cedex 9, France

Paper presented at the de Gennes Discussion Conference, February 2009, Chamonix, France

\begin{abstract}
Rheological model of fluids involving Brownian relaxation, reptation, diffusion, and scission-recombination processes as relaxation mechanisms is formulated. Numerical solution of a particular example of the model displays the S-shape form of the shear rate versus shear stress curves observed in wormlike micellar solutions.
\end{abstract}

\section{Introduction}

An imposed flow affects in ordinary polymeric fluids only the conformation of polymer chains. The molecular weight distribution remains unchanged. In fluids composed of wormlike micelles (sometimes called living polymers), also the molecular weight distribution evolves in time. The rheology of such fluids is thus influenced by both the chain conformation dynamics and the molecular weight dynamics. One of the important manifestation of the complex rheology of such fluids is the appearance of shear banding ( V. Schmitt et al. (1994), J.-F. Berret et al. (1997), J.-B. Salmon et al. (2003))

The physics of fluids composed of wormlike micelles has been discussed extensively on the level of kinetic theory by Cates (1987). Inspired by his physical insight, we formulate a rheological model on a mesoscopic level. The physics

*corresponding author: e-mail: miroslav.grmela@polymtl.ca 
expressed in the model includes the reptation (diffusion along the backbone of the chains), the constraint release due to the dependence of the nonaffine advection of the chains on their length, the flexibility of the chains, diffusion due to spatial inhomogeneities, and the scission-recombination process of the wormlike micelles. The mesoscopic model is constructed as a particular realization of the GENERIC structure. In this way we guarantee that the solutions of the governing equations agree with the experimental observation of the approach to equilibrium of externally unforced fluids and the observation that at these equilibrium states the behavior of the fluids is found to be well described by classical equilibrium thermodynamics.

As for the rheological consequences of the model, we explore only three reduced versions of the model. The first one, investigated previously in Eslami and Grmela (2008), omits the nonaffine advection, the constraint release, the spatial inhomogeneities, and the scission-recombination process. The second is the mesoscopic model, also inspired by Cates (1987), developed recently in Vazquez et al. (2007) and Zhou et al. (2008). In their considerations, the wormlike micelles are not seen as chains of different length but as (Hookean) dumbbells of two types. One type (type $\mathcal{A}$ ) has a fixed length $l$ and the other type (type $\mathcal{B}$ ) the length $l / 2$. The molecular weight distribution dynamics consists of only two processes: breaking a dumbbell $\mathcal{A}$ into two dumbbells $\mathcal{B}$ and reforming a dumbbell $\mathcal{A}$ from two dumbbells $\mathcal{B}$. The third reduced model whose rheological predictions we explore is an extension of the Vazquez et al. (2007) model. Instead of two types of strands we consider three. The third one, denoted $\mathcal{C}$, arises by attaching the two strands $\mathcal{A}$ in a fixed angle.

Our attention in the investigation of the rheological consequences of the models is focused on the appearance of the S-shape form of the shear rate versus shear stress curves indicating shear banding. A very direct mesoscopic rheological model exhibiting this type of curves has been introduced in Bautista et al. (2000), Manero et al. (2007). We formulate a similar model as a particular realization of GENERIC and investigate its rheological consequences.

The governing equations will be constructed in Section 3 as a particular realization of the framework GENERIC. We shall therefore briefly recall it.

Let $x$ denote the state variable, the time evolution of $x$ is governed by

$$
\frac{d x}{d t}=L \Phi_{x}-\frac{\partial \Xi}{\partial \Phi_{x}}
$$

where $\Phi(x, T)$ is the free energy, $T$ is the temperature (kept constant), we use the shorthand notation $\Phi_{x}=\frac{\partial \Phi}{\partial x}, L$ is an operator (Poisson bivector) expressing kinematics of $x\left(\{A, B\}=<A_{x}, L B_{x}>\right.$, where $\{$,$\} is a Poisson bracket, <,>$ is scalar product), $A$ and $B$ are sufficiently regular real valued functions of $x$, $\Xi\left(\Phi_{x}\right)$ is a real valued function of $\Phi_{x}$, called dissipation potential, satisfying: (i) $\Xi$ reaches its minimum at 0 , (ii) $\Xi(0)=0, \Xi$ is convex in a neighborhood of 0 ,

Solutions to (1) have the following properties: $\frac{d \Phi}{d t} \leq 0, x \rightarrow x_{e q}$ as $t \rightarrow \infty$ where $x_{e q}$ is the equilibrium state that is the state at which the free energy $\Phi(x)$ reaches its minimum. These properties express mathematically agreement of solutions to (1) with the experimentally observed compatibility with equilibrium 
thermodynamics (i.e. the observation that fluids that are left undisturbed reach eventually a state, called an equilibrium state, at which its behavior is found to be well described by equilibrium thermodynamics). The Poisson structure of $L$ is, from the physical point of view, an expression of the presence of Newtonian mechanics in the governing equations. It expresses thus a compatibility of (1) with classical mechanics.

To construct a particular realization of (1) means to express the physics of the particular fluid under consideration in $(x, L, \Xi, \Phi)$. We shall now proceed to do it for fluids composed of wormlike micelles.

Equation (1) is essentially an abstract (i.e. applicable on any level of description) and an appropriate (i.e. certain physically important properties of solutions are guaranteed) combination of nondissipative Hamiltonian time evolution (governed by the first term on the right hand side of (1)) and dissipative Ginzburg-Landau type time evolution (generated by the second term on the right hand side of (1)). The Hamiltonian structure of the nondissipative part has been discovered first in the context of hydrodynamics by Clebsch (1895). Equations of the type (1) have started to appear in Dzyaloshinskii and Volovick (1980) and later in Grmela (1984), Kaufman (1984), Morrison (1984). In the form (1) and with the name GENERIC, the abstract time evolution equation (1) has appeared first in Grmela and Ottinger (1997) and Ottinger, Grmela (1997). GENERIC has been further developed in Grmela (2002), (2005), (2010) and in a different direction in Ottinger (2005). As for applications, there is now a large list of new and very useful time evolution equations of complex fluids that have been introduced first as particular realizations of (1).

\section{Model}

The physics that we shall express in (1) is inspired by the physics discussed in Cates (1987) on the level of kinetic theory (see also Section 2.6 below). First, we specify $x, L, \Xi, \Phi$ and then, in Section 2.5 , we write down with them the governing equations (1).

\subsection{State variables, $\mathrm{x}$}

The wormlike micelles are seen as chains of length $l ; 0<l<\infty$. We therefore choose the following state variables

$$
\begin{aligned}
x= & (\mathbf{u}(\mathbf{r}), n(\mathbf{r}, l), \mathbf{c}(\mathbf{r}, s, l)) \\
& 0<l<\infty ; 0 \leq s \leq l ; \int d \mathbf{r} \int_{0}^{\infty} \ln (\mathbf{r}, l) d l=\rho_{p} ;
\end{aligned}
$$

where $\boldsymbol{u}(\boldsymbol{r})$ is the overall momentum of the fluid, $n(\boldsymbol{r}, l)$ is the number of chains of the length in the interval $(l, l+d l)$ in the space interval $(\boldsymbol{r}, \boldsymbol{r}+d \boldsymbol{r}) ; \rho_{p}$ is the total overall arc concentration of wormlike micelles (considered to be a constant). By $\boldsymbol{c}(\boldsymbol{r}, l, s)$ we denote the second moment in the vector $\boldsymbol{Q}$ of the distribution function $\psi(\boldsymbol{r}, \boldsymbol{Q}, l, s)$, where $\boldsymbol{Q}$ is the tangent vector to the chain of 
the length $l ; 0<l<\infty$ at the chain coordinate $s ; 0<s<l$ at the position coordinate $\boldsymbol{r}$. The wormlike micelles are regarded as chains that only locally look as Hookean or other type of dumbbells.

\subsection{Kinematics, $\mathrm{L}$}

As in Eslami and Grmela (2008), we consider the kinematics of $\boldsymbol{u}$ to be the standard kinematic of the fluid momentum field (Marsden and Weinstein (1982)) and we assume that the tensor field $\boldsymbol{c}$ is passively advected (in other words, Lie dragged) by $\boldsymbol{u}$ (i.e. $\boldsymbol{c}$ follows passively the motion of the fluid without influencing it). In addition, we now assume that scalar field $n$ (that is absent in Eslami and Grmela (2008)) is also passively advected by $\boldsymbol{u}$. Consequently, the kinematics of (2) is expressed in the following Poisson bracket:

$$
\begin{aligned}
\{A, B\}= & \int d \mathbf{r} u_{i}\left[\partial_{j}\left(A_{u_{i}}\right) B_{u_{j}}-\partial_{j}\left(B_{u_{i}}\right) A_{u_{j}}\right] \\
& +\int d \mathbf{r} \int_{0}^{\infty} d l n\left[\partial_{j}\left(A_{n}\right) B_{u_{j}}-\partial_{j}\left(B_{n}\right) A_{u_{j}}\right] \\
& +\int d \mathbf{r} \int_{0}^{\infty} d l \int_{0}^{l} d s\left[c_{i j}\left(\partial_{m}\left(A_{c_{i j}}\right) B_{u_{m}}-\partial_{m}\left(B_{c_{i j}}\right) A_{u_{m}}\right)\right. \\
& +c_{k i}\left(A_{c_{i m}} \partial_{k}\left(B_{u_{m}}\right)-B_{c_{i m}} \partial_{k}\left(A_{u_{m}}\right)\right. \\
& +c_{k m}\left(A_{c_{i m}} \partial_{k}\left(B_{u_{i}}\right)-B_{c_{i m}} \partial_{k}\left(A_{u_{i}}\right)\right]
\end{aligned}
$$

We have used above and will use hereafter the summation convention (summation over repeated indices). By the symbol $\partial_{j}$ we denote $\frac{\partial}{\partial r_{j}}$. The symbols $A$ and $B$ stand for arbitrary real valued and sufficiently regular functions of the state variables (2). As in (1) we use the shorthand notation $A_{c_{i j}}=\frac{\partial A}{\partial c_{i j}} ; A_{u_{j}}=\frac{\partial A}{\partial u_{j}}, \ldots$ . The first line corresponds to the standard kinematic of $\boldsymbol{u}$, the second line to the passive advection of the scalar field $n$, and the last three lines to the passive advection of the tensor field $\boldsymbol{c}$.

The physics that is behind the advection is the interaction of the fluid with an obstacle. In our case the obstacle is the molecular chain. The microhydrodynamic formulation of this problem is known as the Stokes problem. The passive advection expressed above in the Poisson bracket (3) corresponds to an approximative solution to the Stokes problem in which the flow is unperturbed by the obstacle. In order to implement a more realistic advection into the kinematics (i.e. into a modified Poisson bracket) we have to in a way include the Stokes problem into the kinematics. This has been done in Gu and Grmela (2008). As an approximation, the active advection turns into the Gordon-Showalter (1972) modification of the passive advection. The slip coefficient arising in the modification will be denoted by the symbol $\zeta$. The passive advection corresponds to $\zeta=0$. The modified advection as well as the correspondingly modified stress tensor are shown below in the governing equations (26) and (27). 


\subsection{Dissipation, $\Xi$}

We shall consider the following five relaxation mechanisms:

(1) Brownian relaxation

generated by the thermodynamic force

$$
X_{i j}^{(B)}(\boldsymbol{r}, l, s)=\Phi_{c_{i j}(r, l, s)}
$$

(2) Reptation

generated by the force

$$
X_{i j}^{(r e p t)}(\boldsymbol{r}, l, s)=\left(X_{i j}^{(B)}(r, l, s)\right)_{s}
$$

(3) Scission-recombination

generated by the force

$$
X^{(s c r)}(\boldsymbol{r}, l)=-\Phi_{n(r, l)}+\Phi_{n\left(r, l^{\prime}\right)}+\Phi_{n\left(r, l^{\prime \prime}\right)}
$$

(4) Diffusion of $n$

generated by the force

$$
X_{i}^{(d i f n)}(\boldsymbol{r}, l)=\partial_{i}\left(\Phi_{n(r, l)}\right)
$$

(5) Diffusion of $\boldsymbol{c}$

generated by the force

$$
X_{i}^{(d i f c)}(\boldsymbol{r}, l, s)=\partial_{k}\left(X_{k i}^{(B)}(\boldsymbol{r}, l, s)\right)
$$

We note that all these five forces disappear at equilibrium (i.e. at states at which the free energy reaches its minimum). The force $X^{(B)}$ is the standard dissipative force used in mesoscopic dynamics of suspensions of dumbbells. As we shall see below, the dissipative term in the time evolution equation that it generates is identical to the dissipative terms derived without use of the free energy (see for example Bird et al. (1987)). According to de Gennes (1971), the reptation motion is a diffusion along the backbone of the chain. The scissionrecombination process is regarded as a chemical reaction $\mathcal{L} \leftrightarrow \mathcal{L}^{\prime}+\mathcal{L}^{\prime \prime} ; l=l^{\prime}+l^{\prime \prime}$, where $\mathcal{L}$ denotes chains of length $l, \mathcal{L}^{\prime}$ chains of length $l^{\prime}$, and $\mathcal{L}^{\prime \prime}$ chains of length $l^{\prime \prime}$. The force $X^{(s c r)}$ is the chemical affinity corresponding to this reaction. The forces $X^{(d i f n)}$ and $X^{(d i f c)}$ are standard forces (spatial gradients of the thermodynamical conjugates of $n$ and $\boldsymbol{c}$, i.e. spatial gradients of derivatives of the free energy with respect to $n$ and $\boldsymbol{c}$ ) generating diffusion.

From the five thermodynamic forces introduced above we now construct the dissipation potential

$$
\Xi=\Xi^{(B r e p t)}+\Xi^{(s c r)}+\Xi^{(d i f)}
$$

The first term on the right hand side of (9) combines the Brownian relaxation with reptation. If we restrict ourselves to states for which the forces $X^{(B)}$ and 
$X^{(r e p t)}$ are not too large (recall that these forces equal zero at equilibrium) then we can neglect all higher order that quadratic terms and choose

$$
\Xi^{(B r e p t)}=\int d \mathbf{r} \int_{0}^{\infty} d l \int_{0}^{l} d s\left(\begin{array}{cc}
X_{i j}^{(B)} & X_{i j}^{(r e p t)}
\end{array}\right) \Lambda_{i j k l}^{(B r e p t)}\left(\begin{array}{c}
X_{k m}^{(B)} \\
X_{k m}^{(r e p t)}
\end{array}\right)
$$

with kinetic coefficients (material parameters)

$$
\Lambda_{i j k m}^{(B r e p t)}=\frac{1}{2}\left(\begin{array}{cc}
\Lambda_{i j k m}^{(B)} & 0 \\
0 & \Lambda_{i j k m}^{(r e p t)}
\end{array}\right)
$$

that we choose, following Eslami and Grmela (2008), in the form

$$
\begin{aligned}
\Lambda^{(B)} & =\Lambda_{0}^{(B)}\left(\kappa_{1} \widehat{c \delta}+\kappa_{2} \widehat{c c}\right) \\
\Lambda^{(r e p t)} & =\Lambda_{0}^{(r e p t)}\left(\kappa_{1} \widehat{c \delta}+\kappa_{2} \widehat{c c}\right)
\end{aligned}
$$

The symbol $`$ means symmetrization. We see easily that the general properties required from the dissipation potential (see Introduction) are satisfied provided $\Lambda^{(B r e p t)}$ is a positive definite matrix. In general, we can also put into (11) off diagonal terms and introduce in this way a coupling of the Brownian relaxation to reptation.

The second term on the right hand side of (9) corresponds to the scissionrecombination process. We use the standard form (see e.g. Grmela (1993))

$$
\begin{aligned}
\Xi^{(s c r)}= & \int_{0}^{\infty} d l \int_{0}^{\infty} d l^{\prime} \int_{0}^{\infty} d l^{\prime \prime} W\left(n ; l, l^{\prime}, l^{\prime \prime}\right) \\
& \times\left(e^{\frac{1}{2} X^{(s c r)}\left(l, l^{\prime}, l^{\prime \prime}\right)}+e^{-\frac{1}{2} X^{(s c r)}\left(l, l^{\prime}, l^{\prime \prime}\right)}-2\right) \\
& W\left(n ; l, l^{\prime}, l^{\prime \prime}\right) \geq 0 \text { the equality holds if } l^{\prime}+l^{\prime \prime} \neq l
\end{aligned}
$$

By $W$ we denote the rate coefficient for the chemical reaction $\mathcal{L} \leftrightarrow \mathcal{L}^{\prime}+\mathcal{L}^{\prime \prime}$. The first requirement in the last line of (13) guarantees satisfaction of the general properties required from the dissipation potential (listed in Introduction) and the second requirement guarantees satisfaction of the constraint formulated in the last equality in the second line of (2).

Finally, $\Xi^{(d i f)}$ combines the diffusion of $n$ with diffusion of $\boldsymbol{c}$. Again, if we neglect higher order than quadratic terms in the diffusion forces, the dissipation potential $\Xi^{(\text {diff) }}$ becomes

$$
\Xi^{(d i f)}=\int_{0}^{\infty} d l \int_{0}^{l} d s\left(\begin{array}{ll}
X_{i}^{(d i f n)} & X_{i}^{(d i f c)}
\end{array}\right) \Gamma_{i j}^{(d i f)}\left(\begin{array}{c}
X_{j}^{(d i f n)} \\
X_{j}^{(d i f c)}
\end{array}\right)
$$

where the matrix $\Gamma^{(d i f)}$ is the matrix of diffusion coefficients (material parameters)

$$
\Gamma_{i j}^{(d i f)}=\frac{1}{2}\left(\begin{array}{ll}
\Gamma_{i j}^{(11)} & \Gamma_{i j}^{(12)} \\
\Gamma_{i j}^{(12)} & \Gamma_{i j}^{(22)}
\end{array}\right)
$$


The positive definitness of $\Gamma^{(d i f)}$ guarantees the general properties required from the dissipation potential.

\subsection{Free energy, $\Phi$}

The physical insight introduced in Cates (1987) and Vazquez et al.(2007) and Zhou et al. (2008) suggests the following choice of the free energy:

$$
\Phi=\Phi^{(u)}+\Phi^{(c)}+\Phi^{(l)}
$$

The first term is the kinetic energy of the overall fluid motion

$$
\Phi^{(u)}=\frac{1}{V} \int d \mathbf{r} \frac{u^{2}}{2 \rho}
$$

where $\rho$ is the mass density (considered to be a constant) of the fluid and $V$ is the volume.

The second term in (16) is the free energy of the chains

$$
\Phi^{(c)}=\frac{1}{V} \int d \mathbf{r} \int_{0}^{\infty} d l \int_{0}^{l} d s n(l)\left(H \operatorname{trc}+K \operatorname{tr}\left(c_{s} c_{s}\right)-\frac{1}{2} k_{B} T \ln \operatorname{det} c\right)
$$

The first term on the right hand side of (18) represents the energy of the local Hookean dumbbell, $H$ is the elastic modulus. It is easy to replace Hookean dumbbells with, say, FENEP dumbbells or rigid rods. The corresponding expression for the free energy can be found for example in Eslami and Grmela (2008). The second term on the right hand side of (18) expresses the bending energy of the chain, $K$ is the bending modulus. The third term on the right hand side of (18) is the entropy contribution to the free energy, $k_{B}$ is the Boltzmann constant, $T$ is the temperature (assumed to be a constant).

The third term in (16) is the contribution to the free energy due to the polydispersity of the length of the chains.

$$
\Phi^{(l)}=\frac{1}{V} \int d \mathbf{r} \int_{0}^{\infty} d l n(l)(\ln n(l)+C(l))
$$

The function $C(l)$ is a material parameter.

\subsection{Governing equation}

We are now in position to write down the governing equations (1). First, we collect the derivatives of the free energy.

$$
\begin{aligned}
\Phi_{u_{i}(r)} & =\frac{u_{i}}{\rho}=v_{i} \\
\Phi_{n(r, l)} & =\ln n+1+C+\int_{0}^{l} d s\left(H \operatorname{trc}+K \operatorname{tr}\left(c_{s} c_{s}\right)-\frac{1}{2} k_{B} T \ln \operatorname{det} c\right) \\
\Phi_{c_{i j}(r, l, s)} & =n(l)\left(H \delta_{i j}-2 K c_{s s}-\frac{1}{2} k_{B} T c_{i j}^{-1}\right)
\end{aligned}
$$


and derivatives of the dissipation potential

$$
\begin{aligned}
\frac{\partial \Xi}{\partial \Phi_{n(r, l)}} & =\frac{\partial \Xi^{(d i f f)}}{\partial \Phi_{n(r, l)}}+\frac{\partial \Xi^{(s c r)}}{\partial \Phi_{n(r, l)}} \\
\frac{\partial \Xi}{\partial \Phi_{c_{i j}(r, s, l)}} & =\frac{\partial \Xi^{(d i f f)}}{\partial \Phi_{c_{i j}(r, s, l)}}+\frac{\partial \Xi^{(B r e p t)}}{\partial \Phi_{c_{i j}(r, s, l)}}
\end{aligned}
$$

where

$$
\begin{aligned}
& \frac{\partial \Xi^{(d i f)}}{\partial \Phi_{n(r, l)}}=-\partial_{k}\left(\int d r^{\prime} \int_{0}^{\infty} d l^{\prime} \frac{\partial \Xi^{(d i f)}}{\partial X_{l}^{(d i f n)}\left(r^{\prime}, l^{\prime}\right)} \frac{\partial X_{l}^{(d i f n)}\left(r^{\prime}, l\right)}{\partial\left(\partial_{k} \Phi_{n(r, l)}\right)}\right) \\
& =-\partial_{k}\left(\Gamma_{k m}^{(11)} \partial_{m}\left(\Phi_{n(r, l)}\right)+\int_{0}^{l} d s \Gamma_{k m}^{(12)} \partial_{i}\left(\Phi_{c_{i m}(r, l, s)}\right)\right) \\
& \frac{\partial \Xi^{(s c r)}}{\partial \Phi_{n(r, l)}}=\int d l^{\prime} \int d l^{\prime \prime} \int d l^{\prime \prime \prime} \frac{\partial \Xi^{(s c r)}}{\partial X^{(s c r)}\left(l^{\prime}, l^{\prime \prime}, l^{\prime \prime \prime}\right)} \frac{\partial X^{(s c r)}\left(l^{\prime}, l^{\prime \prime}, l^{\prime \prime \prime}\right)}{\partial \Phi_{n(r, l)}} \\
& =\frac{1}{2}\left[-\int d l^{\prime \prime} \int d l^{\prime \prime \prime} W\left(n ; l, l^{\prime \prime}, l^{\prime \prime \prime}\right)\left(\frac{1}{2} X^{(s c r)}\left(l, l^{\prime \prime}, l^{\prime \prime \prime}\right)-e^{-\frac{1}{2} X^{(s c r)}\left(l, l^{\prime \prime}, l^{\prime \prime \prime}\right)}\right)\right. \\
& +\int d l^{\prime} \int d l^{\prime \prime \prime} W\left(n ; l^{\prime}, l, l^{\prime \prime \prime}\right)\left(\frac{1}{2} X^{(s c r)}\left(l^{\prime}, l, l^{\prime \prime \prime}\right)-e^{-\frac{1}{2} X^{(s c r)}\left(l^{\prime}, l, l^{\prime \prime \prime}\right)}\right) \\
& \left.+\int d l^{\prime} \int d l^{\prime \prime} W\left(n ; l^{\prime}, l^{\prime \prime}, l\right)\left(^{\frac{1}{2} X^{(s c r)}\left(l^{\prime}, l^{\prime \prime}, l\right)}-e^{-\frac{1}{2} X^{(s c r)}\left(l^{\prime}, l^{\prime \prime}, l\right)}\right)\right] \\
& \frac{\partial \Xi^{(d i f)}}{\partial \Phi_{c_{i j}(r, s, l)}}=-\frac{1}{2}\left[\partial_{i}\left(\Gamma_{j m}^{(11)} \partial_{k}\left(\Phi_{c_{k m}(r, s, l)}\right)+\Gamma_{j m}^{(12)} \partial_{m}\left(\Phi_{n(r, l)}\right)\right)\right. \\
& \left.+\partial_{j}\left(\Gamma_{i m}^{(11)} \partial_{k}\left(\Phi_{c_{k m}(r, s, l)}\right)+\Gamma_{i m}^{(12)} \partial_{m}\left(\Phi_{n(r, l)}\right)\right)\right] \\
& \frac{\partial \Xi^{(B r e p t)}}{\partial \Phi_{c_{i j}(r, s, l)}}=\Lambda_{i j k m}^{(B)} \Phi_{c_{k m}(r, s, l)}-\left(\Lambda_{i j k m}^{(r e p t)}\left(\Phi_{c_{k m}(r, s, l)}\right)_{s}\right)_{s}
\end{aligned}
$$

The time evolution equations (1) become:

$$
\begin{aligned}
\frac{\partial u_{i}(\boldsymbol{r})}{\partial t}= & -\partial_{j}\left(u_{i} \Phi_{u_{j}}\right)-\partial_{j} p-\partial_{j} \sigma_{i j} \\
\frac{\partial n(\boldsymbol{r}, l)}{\partial t}= & -\partial_{j}\left(n \Phi_{u_{j}}\right)-\frac{\partial \Xi}{\partial \Phi_{n(r, l)}} \\
\frac{\partial c_{i j}(\boldsymbol{r}, s, l)}{\partial t}= & -\partial_{k}\left(c_{i j} \Phi_{u_{k}}\right)+c_{i k}\left(\partial_{k} \Phi_{u_{j}}-\zeta D_{j k}\right)+c_{j k}\left(\partial_{k} \Phi_{u_{i}}-\zeta D_{i k}\right) \\
& -\frac{\partial \Xi}{\partial \Phi_{c_{i j}(r, s, l)}}
\end{aligned}
$$

where $D_{i k}=1 / 2\left(\partial_{k} \Phi_{u_{i}}+\partial_{i} \Phi_{u_{k}}\right), p$ is the scalar pressure that is determined by the incompressibility requirement and the extra stress tensor $\boldsymbol{\sigma}$ is given by

$$
\sigma_{i j}(r)=-\int_{0}^{\infty} d l \int_{0}^{l} d s(1-\zeta) c_{i k}(\boldsymbol{r}, s, l) \Phi_{c_{i j}(r, s, l)}
$$


The expression (27) for the extra stress tensor arises automatically as a part of writing down explicitly the time evolution equation (1). In particular, we emphasize that we have arrived at the expression (27) without using any additional physical considerations than those that lead us to $L$ (see Section 2.2) and that the expression (27) is guaranteed to be compatible with the time evolution equations of the internal structure (i.e. with the time evolution of $\boldsymbol{c}(\boldsymbol{r}, s, l)$ and $n(\boldsymbol{r}, l)$.

The material parameters entering the governing equations (26) are:

$$
\text { material parameters } \equiv\left(\zeta, H, K, C, \Lambda^{(B r e p t)}, W, \Gamma^{(d i f)}\right)
$$

where $\zeta$ has arisen in the advection, $(H, K, C)$ have arisen in the free energy (16) ( $H$ is the local elastic modulus, $K$ is the bending modulus and $C(l)$ is the parameter appearing in (19)), and $\left(\Lambda^{(B r e p t)}, W, \Gamma^{(\text {dif })}\right)$ are kinetic coefficients arising in the formulation of dissipative processes (local Brownian relaxation, reptation, scission-recombination, and diffusion)

\subsection{Physics expressed in Eq.(26)}

Before discussing further the material parameters (28) and solutions of (26), we recall the physics on which Eqs.(26) are based. The physics is not new. It is the physics that has gradually arisen in the one chain kinetic theory of polymeric fluids. What is new is that we are expressing it on a more macroscopic (mesoscopic) level of description and with the use of the GENERIC framework. The mesoscopic level on which we are placing ourselves has its advantages and disadvantages. Among the advantages we mention the relative mathematical simplicity of the governing equations (the passage to mesoscopic formulations can be regarded as a step in the preparation of kinetic equations for the process of solving them) and the possibility of broader interpretation and broader applicability of the physics introduced in the kinetic theory. The most visible advantage of the GENERIC formulation is that the expression for the extra stress tensor in terms of the microstructural state variables arises automatically as a part of the formulation and is guaranteed to be compatible with the equations governing the time evolution of the microstructure. Indeed, any new physics introduced into the time evolution of the microstructure changes, in general, forces inside the fluid and thus the expression for the extra stress tensor $\boldsymbol{\sigma}$. The GENERIC formalism guarantees that the expression for $\boldsymbol{\sigma}$ arising in the process of constructing the model takes the new physics into account. As for the disadvantages of more macroscopic formulations, it is mainly the impossibility to express details. As in any reduction of microscopic theories, the principal challenge is to recognize which details are important and which are not for determining macroscopic properties of our direct interest. The mesoscopic reformulation of the one chain kinetic theory has started in Eslami, Grmela (2008). The completeness of the reformulation has not been the objective (see the section titled "Relation to the level $\mathcal{L}_{1 k t}$ " in Eslami, Grmela (2008)). Below, we shall list some of the elements of the physics introduced on the level of one 
chain kinetic theory that have and have not been expressed in the preceding section.

\section{Tube constraint}

How do we express the presence of other particles in the one particle kinetic theory in which one particle distribution function serves as the microstructural state variable? In the classical kinetic theories of gases it is done by the concept of a mean field, i.e. by an appropriately averaged force with which the surrounding particles influence the motion of the one particle represented by the one particle distribution function. In the one chain kinetic theory it is done (see Edwards (1967)) by a fictitious tube constraint formed around the chain represented by the one chain distribution function. Inside the tube, the chain moves predominantly along its backbone (de Gennes (1971)). This motion, called a reptation motion by de Gennes, is expressed in the mesoscopic formulation by the diffusion, generated by the force (5), in the chain coordinate $s$. The reptation motion is the primary relaxation mechanism in our formulation. Since it is the tube that causes the chains to make the reptation motion and since it is only by making this type of motion that the chain can escape from the tube, the primary relaxation mechanism can also be interpreted as a tube escape mechanism.

\section{Nonaffine advection and constraint release}

The tube itself evolves in time. In particular, due to the relative motion of chains caused by the dependence of the nonaffine advection and diffusion on some properties (as for instance the length) of chains, the tube may disappear and later reappear. This additional perturbative mechanism that enhances the relaxation has been called, in the context of the one chain kinetic theory, a constraint release. This process can be taken into account either by introducing a new microstructural state variable characterizing the tube and a new equation governing its time evolution, or by letting the kinetic coefficients that arise in the formulation of the dissipation, as well as the coefficient representing the slip in the advection, depend in an appropriate way on the chain distribution function. It is the latter strategy that was used to express the constraint release in Marrucci (1996) and Cates (1987). In the GENERIC formulation presented above, the slip coefficient is $\zeta$ and the kinetic coefficients are introduced in the dissipation potential. Following Marrucci and Cates we can, in principle, express the physics of the constraint release in an appropriate choice of the dependence of the material parameters (28) on the state variables. Since in this paper we shall investigate rheological consequences of only a very reduced version of (26), we shall not attempt to do it. The constraint release is also not discussed in the mesoscopic formulation developed in Eslami and Grmela (2008).

\section{Chain flexibility}

The energy associated with bending the chains is included into the free en- 
ergy (see the second term in (18)). This element of the physics of polymer chains is often ignored in the kinetic theory formulations.

\section{Spatial inhomogeneity}

Experimental observation of spontaneous formation of spatial inhomogeneities in fluids composed of wormlike micelles subjected to a flow (e.g. shear banding) indicates the pertinence of including into the model the physics associated with the nonuniform dependence on the position coordinate $\boldsymbol{r}$. This has been done in the model of Vazquez et al. (2007) and Zhou et al. (2008). In the mesoscopic formulation developed in the previous section this physics is expressed in the thermodynamic forces (7) and (8).

\section{Scission-recombination}

The chemical-reaction type formulation of the scission-recombination process introduced by Cates (1987) on the level of kinetic theory is reproduced on the mesoscopic level considered in this paper by including into the list of thermodynamic forces that drive the fluid to equilibrium the force (13). As we have already mentioned above, the delicate interplay between the constraint release and the scission recombination, that is discussed in detail in Cates (1987), is not attempted in this paper to be reproduced.

\section{Illustrations}

Now we turn to solutions of Eqs.(26). Even without a further specification of the material parameters (28) we know that solutions to Eqs.(26) agree with experimental observations constituting the basis of equilibrium thermodynamics (i.e. the observation that fluids that are left undisturbed reach a state, called an equilibrium state, at which their behavior is found to be well described by equilibrium thermodynamics). This is because Eqs.(26) have been constructed as a particular realization of (1) and solutions of (1) are guaranteed to posses this property. We are however mainly interested in other types of experimental observations. In particular, in observations of responses of the fluid to an imposed flow. In order to obtain solutions of Eqs.(26) predicting such responses we have to, first, specify the material parameters (28) and, second, solve Eqs.(26) numerically. In this paper we shall restrict ourselves only to a few particular cases of Eqs.(26) The first two recalled in Subsections 3.1 and 3.2 have already been worked out previously. The third one, presented in Section 3.3, is new. A particular attention is payed to rheological predictions indicating the occurrence of shear banding. With this focus in mind and inspired by Bautista et al. (2000), we then identify in Section 3.4 a "minimalist" internal structure model (a particular realization of (1)) predicting the S-shape form of the curve $\sigma_{12}$ versus shear rate that is indicative of shear banding. 


\subsection{Reptation without the scission-recombination process}

We replace the fluid composed of wormlike micelles by a standard polymeric fluid composed of chains with a fixed molecular weight distribution. Moreover, we assume that the microstructure of the fluid is spatially homogeneous. Under these simplifying assumptions, Eqs.(26) reduce to the governed equations developed and investigated in Eslami and Grmela (2008). Their rheological predictions have been found to be in a good agreement with results of experimental observations.

\subsection{Two species scission-recombination process without reptation}

Another particular case of Eqs.(26) is the one discussed in detail in Vazquez et al.(2007) and Zhou et al. (2008). The microstructure of the fluid is considered to be spatially inhomogeneous as in Section 2 but the chains composing the fluid are replaced by two types of dumbbells, type $\mathcal{A}$ and type $\mathcal{B}$. The scissionrecombination process reduces in this particular case to the reaction $\mathcal{A} \leftrightarrow 2 \mathcal{B}$, i.e. a split of the type $\mathcal{A}$ dumbbell into two type $\mathcal{B}$ dumbbells and the inverse process consisting of the reformation of the dumbbell $\mathcal{A}$ from two dumbbells $\mathcal{B}$. Rheological predictions reported in Vazquez et al.(2007) and Zhou et al. (2008) include shear banding.

\subsection{Three species scission-recombination process without reptation}

In this illustration we extend the model introduced and investigated in Vazquez et al.(2007) and Zhou et al. (2008). Instead of two types of strands, we consider three. The first two, denoted $\mathcal{A}$ and $\mathcal{B}$, are the same as in Vazquez et al.(2007) and Zhou et al. (2008). The third, denoted $\mathcal{C}$, is a platelet formed by joining two strands of the type $\mathcal{B}$ in a fixed angle $\varphi ; 0<\varphi<\pi$. The scission-recombination process consists of the following two reactions: $\mathcal{A} \leftrightarrow 2 \mathcal{B}$ and $2 \mathcal{B} \leftrightarrow \mathcal{C}$. The main objective is to show that the results obtained in Vazquez et al.(2007) and Zhou et al. (2008), in particular the results related to the multi-valuedness of the shear rate as a function of shear stress, are "stable" with respect to changes in the scission-recombination dynamics. We shall indeed see that the extension from two to three species of strands does not change qualitatively the results obtained in Vazquez et al.(2007) and Zhou et al. (2008).

We now recall the governing equations arising in Vazquez et al.(2007) and Zhou et al. (2008). By $n_{A}, n_{B}$ we denote the number densities of the species $\mathcal{A}, \mathcal{B}$ and by $c_{A}, c_{B}$ their production rates. The associated conformation tensors are denoted $\mathbb{A}$ and $\mathbb{B}$.

$$
\begin{gathered}
c_{A}=c_{A e q}+\frac{1}{3} \xi\left(\nabla v: \frac{\mathbb{A}}{n_{A}}\right) \\
c_{B}=\text { constant }
\end{gathered}
$$




$$
\begin{gathered}
\mu \frac{\partial n_{A}}{\partial t}=\frac{c_{B}}{2} n_{B}^{2}-c_{A} n_{A} \\
\mu \frac{\partial n_{B}}{\partial t}=-c_{B} n_{B}^{2}+2 c_{A} n_{A} \\
\mu\left(\frac{\partial \mathbb{A}}{\partial t}-\nabla v \cdot \mathbb{A}-\mathbb{A} \cdot \nabla v^{T}\right)+\mathbb{A}-n_{A} I=c_{B} n_{B} \mathbb{B}-c_{A} \mathbb{A} \\
\varepsilon \mu\left(\frac{\partial \mathbb{B}}{\partial t}-\nabla v \cdot \mathbb{B}-\mathbb{B} \cdot \nabla v^{T}\right)+\mathbb{B}-\frac{1}{2} n_{B} I=\varepsilon\left(-2 c_{B} n_{B} \mathbb{B}+2 c_{A} \mathbb{A}\right)
\end{gathered}
$$

The stress tensor is then given by

$$
\sigma=\mathbb{A}+2 \mathbb{B}-\left(n_{A}+n_{B}\right) I+\beta\left(\frac{\nabla v+\nabla v^{T}}{2}\right)
$$

where $I$ is the identity matrix.

We considered the case in which the model parameters are set to: $c_{\text {Aeq }}=0.5 ; c_{B}=1 ; \mu=1 ; \varepsilon=0.005 ; \beta=6.7810^{-5} ; \xi=0.3$.

The initial condition is taken to be: $n_{A}=1 ; n_{B}=1 ; \mathbb{A}=I ; \mathbb{B}=I . n_{B} / 2$. The computed solution is depicted in figure 1 .

The modified (three species) version of this model introduces a new species $\mathcal{C}$ and consequently a new state variable $n_{C}$ and a new production rate $c_{C}$. The associated conformation tensor is denoted $\mathbb{C}$. Since the new species $\mathcal{C}$ is composed of platelets, a lower-convected derivative is used to describe its advection by the flow. The governing equations describing the three species model are the following:

$$
\begin{gathered}
c_{C}=c_{C e q}-\frac{1}{3} \xi\left(\nabla v: \frac{\mathbb{C}}{n_{C}}\right) \\
c_{A}=c_{A e q}+\frac{1}{3} \xi\left(\nabla v: \frac{\mathbb{A}}{n_{A}}\right) \\
c_{B}=\text { constant } \\
\mu \frac{\partial n_{C}}{\partial t}=\frac{c_{B}}{2} n_{B}^{2}-c_{C} n_{C} \\
\mu \frac{\partial n_{A}}{\partial t}=\frac{c_{B}}{2} n_{B}^{2}-c_{A} n_{A} \\
\mu \frac{\partial n_{B}}{\partial t}=-2 c_{B} n_{B}^{2}+2 c_{C} n_{C}+2 c_{A} n_{A} \\
\mu\left(\frac{\partial \mathbb{C}}{\partial t}+\nabla v^{T} \cdot \mathbb{C}+\mathbb{C} \cdot \nabla v\right)+\mathbb{C}-n_{C} I=c_{B} n_{B} \mathbb{B}-c_{C} \mathbb{C} \\
\mu\left(\frac{\partial \mathbb{A}}{\partial t}-\nabla v \cdot \mathbb{A}-\mathbb{A} \cdot \nabla v^{T}\right)+\mathbb{A}-n_{A} I=c_{B} n_{B} \mathbb{B}-c_{A} \mathbb{A}
\end{gathered}
$$




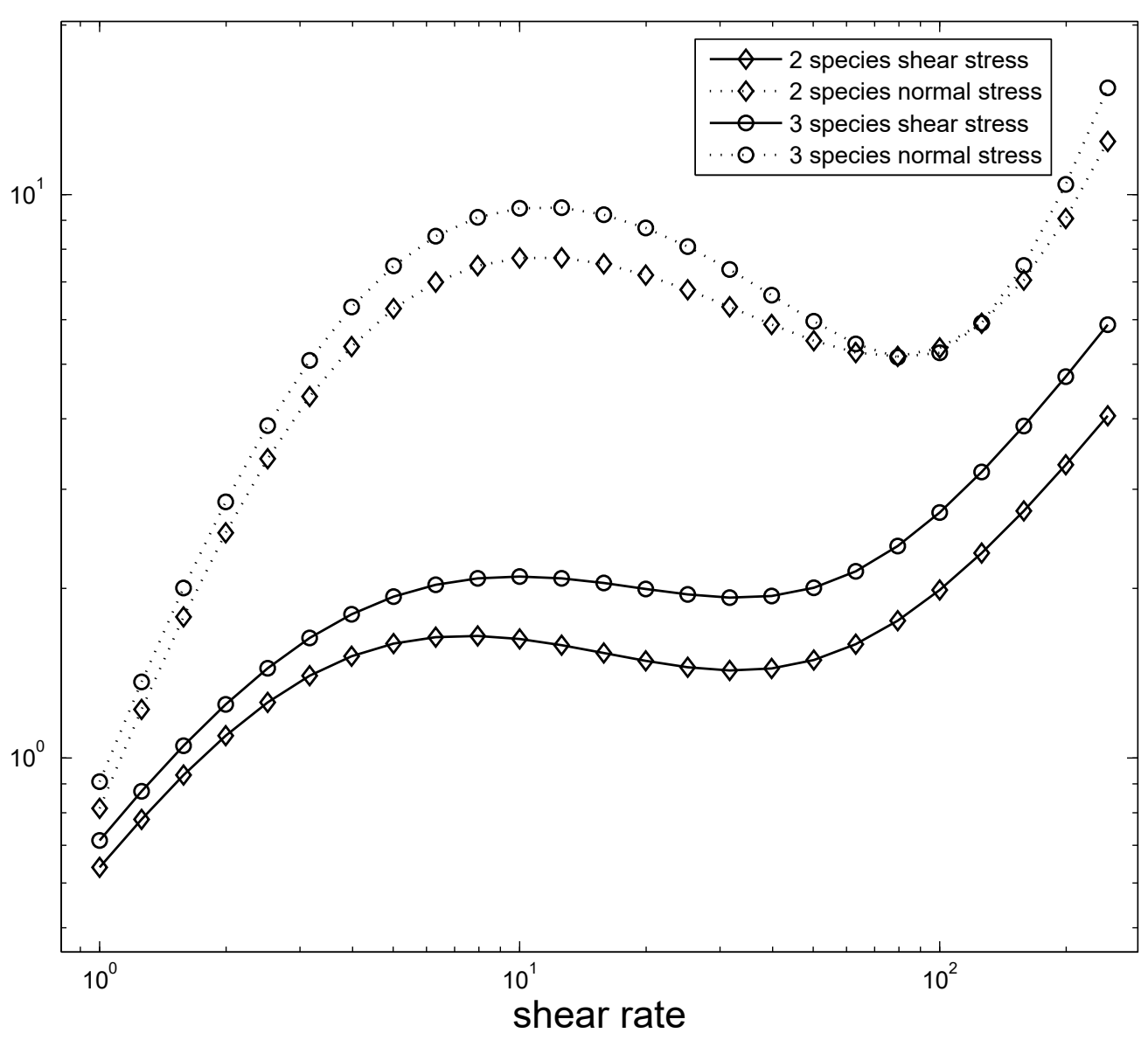

Figure 1: Comparison of the two and three species model

$$
\begin{gathered}
\varepsilon \mu\left(\frac{\partial \mathbb{B}}{\partial t}-\nabla v \cdot \mathbb{B}-\mathbb{B} \cdot \nabla v^{T}\right)+\mathbb{B}-\frac{1}{2} n_{B} I=\varepsilon\left(-2 c_{B} n_{B} \mathbb{B}+2 c_{C} \mathbb{C}+2 c_{A} \mathbb{A}\right) \\
\sigma=-\mathbb{C}+\mathbb{A}+2 \mathbb{B}-\left(-n_{C}+n_{A}+n_{B}\right) I+\beta\left(\frac{\nabla v+\nabla v^{T}}{2}\right)
\end{gathered}
$$

In the numerical example we considered the following set of parameters: $c_{C e q}=0.5 ; c_{A e q}=0.5 ; c_{B}=1 ; \mu=1 ; \varepsilon=0.005 ; \beta=6.7810^{-5} ; \xi=0.3$. The initial condition is taken to be: $n_{C}=0.5 ; n_{A}=0.5 ; n_{B}=1 ; \mathbb{A}=\mathbb{C}=$ $I ; \mathbb{B}=I n_{B} / 2$.

Figure 1 compares the the shear and the normal stress of both models in a simple shear flow. We see indeed that the important property of solution to the governing equations, namely the multi-valuedness of shear rate as a function of shear rate, appears in both two species and three species models. We hope to investigate this property in the context of the general model (26), (27) in a future paper. 


\section{4 "Minimalist" rheological model implying S-shape de- pendence of $\sigma_{12}$ on $\dot{\gamma}$}

Bautista et al. (2000) have noted that a rheological model involving one tensor (the stress tensor in their model) and one scalar as structural state variables (i.e. the type of the model introduced first in Acierno et al. (1976)) can reproduce an S-shape dependence of $\sigma_{12}$ on $\dot{\gamma}$ in a simple shear flow. We shall present below a GENERIC model in which the internal structure is chosen to be characterized by one conformation tensor $\boldsymbol{c}$, characterizing macromolecules regarded as Hookean dumbbells, and one scalar structural variable $\mu$ taking values in the interval $[0,1]$. With quadratic dependence of the mobility of the dumbbell on $\mu$, the model (that we call "minimalist" since it involves a minimum of the microstructure) predicts the S-shape form of the curve $\sigma_{12}$ versus $\dot{\gamma}$.

We choose the state variables to be

$$
x=(\boldsymbol{c}, \mu)
$$

where $\boldsymbol{c}$ is an orientation tensor (a symmetric and positive definite three-bythree tensor) and $\leq 0 \mu \leq 1$ is a dimensionless scalar. For the sake of simplicity we assume that both $\boldsymbol{c}$ and $\mu$ are independent of the position coordinate. There are many physical pictures of the microstructure (e.g. dumbbells or a transient network) that can be discussed in terms of the state variables (46) and thus (46) can have many physical interpretations. We shall now proceed to formulate the model.

Kinematics, L

$$
\begin{aligned}
\{A, B\}= & \int d \boldsymbol{r} u_{i}\left[\partial_{j}\left(A_{u_{i}}\right) B_{u_{j}}-\partial_{j}\left(B_{u_{i}}\right) A_{u_{j}}\right] \\
& +c_{k i}\left(A_{c_{i m}} \partial_{k}\left(B_{u_{m}}\right)-B_{c_{i m}} \partial_{k}\left(A_{u_{m}}\right)\right. \\
& +c_{k m}\left(A_{c_{i m}} \partial_{k}\left(B_{u_{i}}\right)-B_{c_{i m}} \partial_{k}\left(A_{u_{i}}\right)\right. \\
& +2 c_{k l}\left(A_{\mu} \partial_{k}\left(B_{u_{l}}\right)-B_{\mu} \partial_{k}\left(A_{u_{l}}\right)\right.
\end{aligned}
$$

The easiest way to verify that (47) is indeed a Poisson bracket is to make the following observation: We start with state variables $(\boldsymbol{b}, \nu)$ whose kinematics is expressed by the first three lines in (47). There is no contribution from $\nu$ into the bracket. Indeed, since $\nu$ is a scalar that is independent of $\boldsymbol{r}$, it is not affected by the imposed flow. The bracket consisting of the first three lines in (47) is clearly a Poisson bracket since it is a well known bracket expressing kinematics of the orientation tensor. Next, we make a one-to-one transformation $(\boldsymbol{b}, \nu) \leftrightarrow(\boldsymbol{c}, \mu)$ by: $\mu=\nu+\operatorname{tr} \boldsymbol{b} ; \boldsymbol{c}=\boldsymbol{b}$. This transformation then transforms the Poisson bracket expressing the kinematics of $\boldsymbol{b}, \nu$ into the bracket (47). This proves that the bracket (47) is a Poisson bracket.

\section{Dissipation, $\Xi$}


The thermodynamic forces driving (47) to equilibrium are:

$$
\begin{aligned}
& X_{i j}^{c)}=\Phi_{c_{i j}} \\
& X^{(\mu)}=\Phi_{\mu}
\end{aligned}
$$

and the dissipation potential is introduced by

$$
\Xi=\frac{1}{2} X_{i k}^{(c)} \Lambda c_{k l} X_{l i}^{(c)}+\frac{1}{2} \lambda\left(X^{(\mu)}\right)^{2}
$$

In order that this dissipation potential satisfies all the properties required from it (we recall, $\Xi(0)=0, \Xi$ reaches its minimum at 0 , and $\Xi$ is convex in a neighborhood of 0 ), the two kinetic coefficients $\Lambda$ and $\lambda$ can depend on $\mu$ but have to be positive. We shall specify them later.

Free Energy, $\Phi$

Similarly as in Section 2.4, we choose

$$
\Phi=\frac{1}{V} \int d \boldsymbol{r} \frac{u^{2}}{2 \rho}+H \operatorname{tr} \boldsymbol{c}-\frac{G}{2} \ln \left(1-\mu^{2}\right)-\frac{1}{2} k_{B} T \ln \operatorname{det} \boldsymbol{c}
$$

where $H, G$, similarly as in (18), are two elastic moduli (assumed to be independent of $\mu$ ). The first term represents the overall kinetic energy, the second and the third term are contributions of the internal structure to the energy, and the fourth term represents the entropy part of the free energy. We note that in the absence of $\mu$, the free energy is simply a free energy of a Hookean dumbbell. The GENERIC model with this free energy is the same as the Maxwell model used in Bautista et al. (2000). The requirement of the thermodynamic consistency (in other words the requirement that the model is a particular realization of GENERIC (1)) forces us however to depart from the Bautista et al. (2000) model in the time evolution of $\mu$, in its coupling to the time evolution equation of $\boldsymbol{c}$, and in the involvement of $\mu$ in the extra stress tensor. In order to guarantee that $\mu$ remains in a finite interval, we choose the FENEP like potential (the third term in (51)).

We see easily that $\boldsymbol{c}$ at equilibrium (i.e. solutions to $\Phi_{\boldsymbol{c}}=0$ ) is

$$
\left(c_{i j}\right)_{e q}=\frac{k_{b} T}{2 H} \delta_{i j}
$$

and $\mu$ at equilibrium (i.e. solution to $\Phi_{\mu}=0$ ) is

$$
\mu=0
$$

Governing equation 
With the specifications above, (1) becomes:

$$
\begin{aligned}
\frac{d c_{i j}}{d t} & =c_{i k} \partial_{k} v_{j}+c_{j k} \partial_{k} v_{i}-\Lambda\left(H c_{i j}-\frac{k_{B} T}{2} \delta_{i j}\right) \\
\frac{d \mu}{d t} & =2 c_{i j}\left(\partial_{i} v_{j}-\zeta D_{i j}\right)-\frac{2 \lambda G \mu}{1-\mu^{2}} \\
\sigma_{i j} & =-2\left(c_{i k} \Phi_{c_{k j}}+c_{i j}(1-\zeta) \Phi_{\mu}\right) \\
& =-2 c_{i j} H+k_{B} T \delta_{i j}-2(1-\zeta) c_{i j} \frac{2 G \mu}{1-\mu^{2}}
\end{aligned}
$$

\section{Solution}

In the simple shear flow $\partial_{i} v_{j}=0$ except $\partial_{2} v_{1}=\dot{\gamma}$. In the stationary state we have

$$
\begin{aligned}
2 c_{12} \dot{\gamma} & =\Lambda\left(H c_{11}-\frac{k_{B} T}{2}\right) \\
c_{22} \dot{\gamma} & =\Lambda H c_{12} \\
0 & =\Lambda\left(H c_{22}-\frac{k_{B} T}{2}\right) \\
0 & =\Lambda\left(H c_{33}-\frac{k_{B} T}{2}\right) \\
0 & =c_{23} \\
0 & =c_{13} \\
2 c_{12} \dot{\gamma}(1-\zeta) & =\frac{2 \lambda G \mu}{1-\mu^{2}} \\
\sigma_{12} & =-2 c_{12}\left(H+2(1-\zeta) \frac{2 G \mu}{1-\mu^{2}}\right)
\end{aligned}
$$

These equations reduce to:

$$
(\dot{\gamma})^{2} \frac{k_{B} T}{H^{2}} \frac{1-\zeta(\mu)}{\Lambda(\mu)}=2 G \frac{\mu \lambda(\mu)}{1-\mu^{2}}
$$

and

$$
\sigma_{12}=-\dot{\gamma} \frac{k_{B} T}{H^{2}} \frac{1}{\Lambda(\mu)}\left(H+4 G \frac{\mu(1-\zeta(\mu))}{1-\mu^{2}}\right)
$$

Let us assume that the functions $\Lambda(\mu), \lambda(\mu)$, and $\zeta(\mu)$ are given. Equation (56) implies then $\mu(\dot{\gamma})$. If this function is inserted into Eq. $(57)$ we obtain $\sigma_{12}(\dot{\gamma})$.

By choosing $\Lambda=0.01+\mu^{2} ; \lambda=1 ; \zeta=0.98$ we obtain $\mu(\dot{\gamma})$ and $\sigma_{12}(\dot{\gamma})$ depicted on the following two figures (figures 2 and 3 ). 


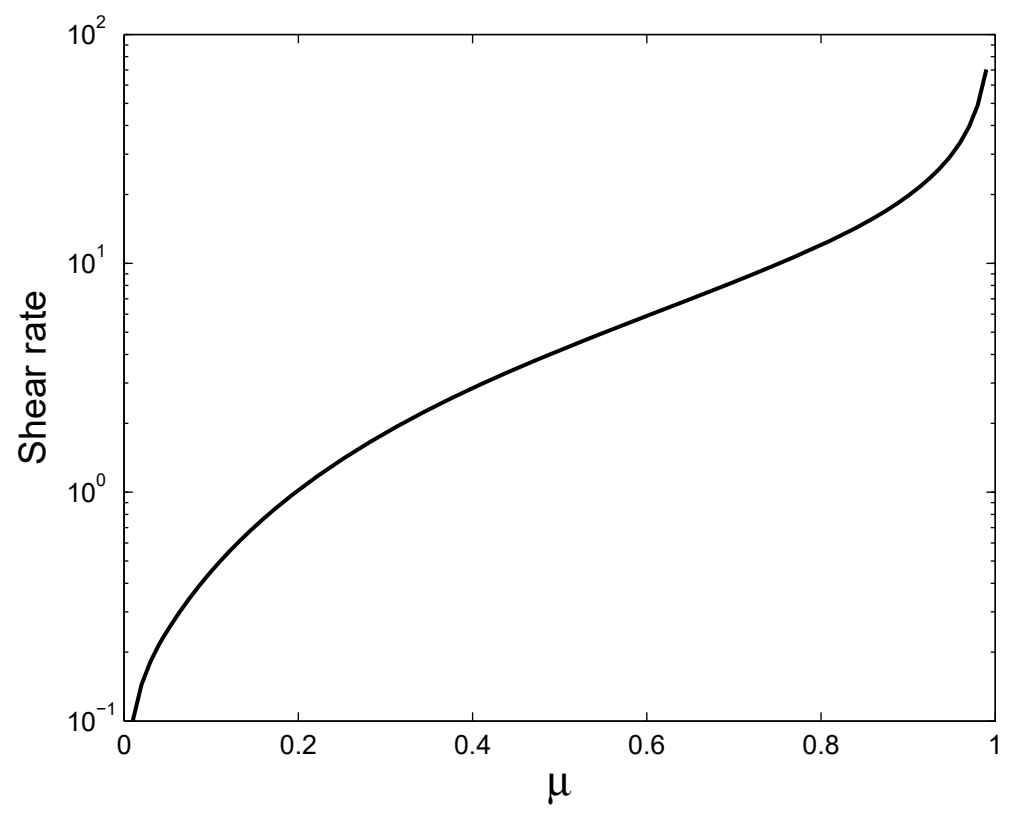

Figure 2: Shear rate versus the parameter $\mu$

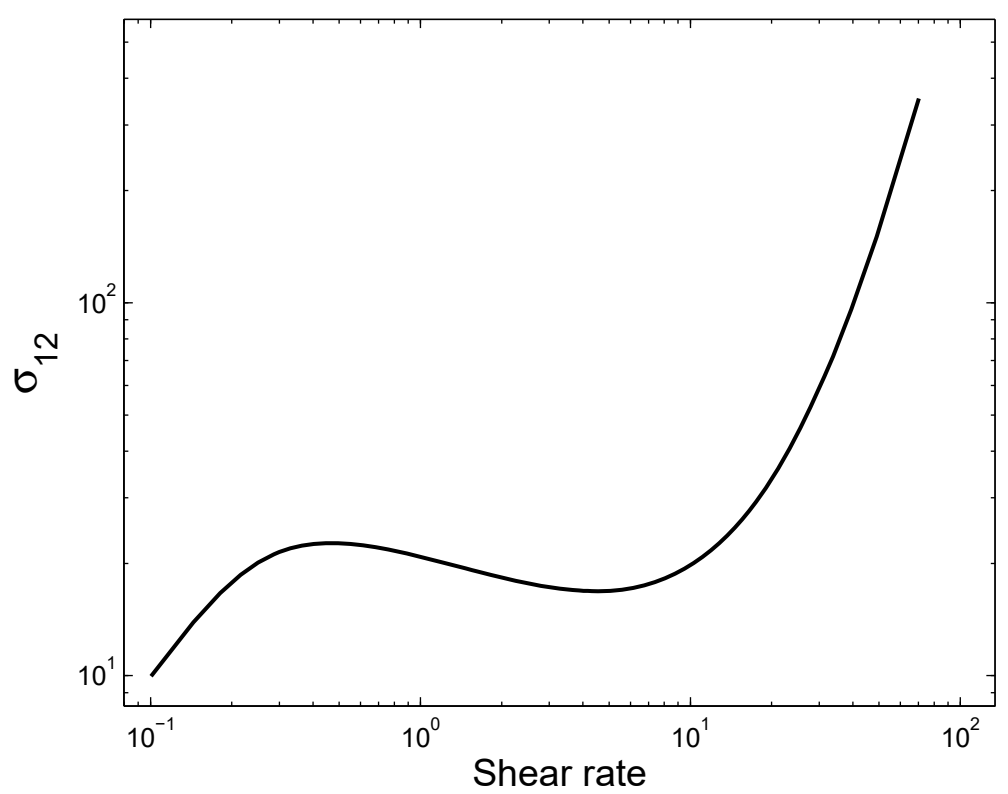

Figure 3: Stress versus shear rate

\section{Concluding remarks}

In this paper we investigate theoretically a fluid composed of chains whose conformation as well as length distribution evolve in time. The relaxation mechanisms include: local Brownian relaxation, reptation, diffusion, and a scissionrecombination process. The mesoscopic formulation of the time evolution is in- 
troduced in Section 2 as a particular realization of GENERIC. The physics that it expresses is motivated by the the physics developed on the level of one chain kinetic theory, in particular then by the physics on which Cates' model (Cates (1987)) is based. Rheological predictions of the mesoscopic model are calculated only for three reduced versions of the model, namely, the model developed by Eslami and Grmela (2008), the model developed in Vazquez et al.(2007), Zhou et al. (2008), and an extension of the model of Vazquez et al.(2007) and Zhou et al. (2008) discussed in Section 3.3. Rheological predictions calculated in Section 3.3 are compared with those reported in Vazquez et al.(2007) and Zhou et al. (2008). A particular attention is payed to the appearance of S-shape form of the shear stress versus shear rate curves that is indicative of the appearance of shear banding. In Section 3.4 we propose a "minimalist" GENERIC model predicting the S-shape.

\section{References}

Acierno D., La Mantia L.P., Marrucci G. and Titomanlio G., A non-linear viscoelastic model with structure-dependent relaxation times: I. Basic formulation, J. Non-Newtonian Fluid Mech. 1, 125 (1976)

Bautista F., Solerto J.F.A., Pérez-López J.H., Puig J.E., Manero O., On the shear banding flow of elongated micellar solutions, J. Non-Newtonian Fluid Mech. 94, 57-66 (2000)

Berret J.-F., Porte G. and Decruppe J.-P., Inhomogeneous shearing flows in wormlike micelles: A master "dynamic phase diagram", Phys. Rev. E 55, 1668-1676 (1997)

Cates M.E., Reptation of living polymers: Dynamics of entangled polymers in the presence of reversible chain-scission reactions, Macromolecules 20 22892296 (1987)

Clebsch A., Ueber die Integration der hydrodynamische Gleichungen, J. Reine Angew. Math, 56 1-10 (1895)

de Gennes P.G., Reptation of polymer chain in the presence of fixed obstacles, J.Chem.Phys. 55 572-579 (1971)

Dzyaloshinskii I.E., Volovick G.E., Poisson brackets in condense matter physics, Ann. Phys. (NY) 125, 67-97 (1980)

Edwards S.F., Proc. Roy. Soc. 92 , 9 (1967)

Eslami H., Grmela M., Mesoscopic formulation of reptation, Rheol. Acta 47, 399-415 (2008)

Gordon R.J., Schowalter W.R., Anisotropic fluid theory: a different approach to the dumbbell theory of dilute polymer solutions, Trans. Soc. Rheol. 16 (1972) 7997.

Grmela M., Particle and Bracket Formulations of Kinetic Equations , Contemporary Math. 28, 125-132 (1984), Physics Letters A 102, 355 (1984)

Grmela M., Thermodynamics of driven systems, Phys. Rev.E, 48, 5689 (1993)

Grmela M., Reciprocity Relations in Thermodynamics, Physica A 309, $304=328(2002)$ 
Grmela M., Geometry of Mesoscopic Dynamics and Thermodynamics, J. NonNewtonian Fluid Mech. 120, 137-147 (2004)

Grmela M., Multiscale Equilibrium and Nonequilibrium Thermodynamics in Chemical Engineering (to appear in Advances in Chemical Engineering (2010))

$\mathrm{Gu}$ J.F. and Grmela M., GENERIC model of active advection, J. NonNewtonian Fluid Mech. 152, 12-26 (2008); Flow properties of immiscible blends: Doi-Ohta model with active advection, Phys. Rev. E 78, 056302 (2008)

Grmela M. and Ottinger H.C., Dynamics and thermodynamics of complex fluids: I Development of a general formalism Phys. Rev. E 55, 6620-6632 (1997)

Kaufman A.N., Dissipative Hamiltonian systems: A unifying principle, Phys. Letters A 100, 419 (1984)

Manero O., Pérez-López J.H., Escalante J.I., Puig J.E., Bautista F., A thermodynamic approach to rheology of complex fluids: The generalized BMP model, J. Non-Newtonian Fluid Mech. 46, 22-29 (2007)

Marrucci G., Dynamics of entanglements: A nonlinear model consistent with the Cox-Merz rule, J. Non-Newtonian Fluid Mech. 62, 279 (1996)

Marsden E., Weinstein A., Physica D 4, 394 (1982)

Morrison P.J., Bracket formulation for irreversible classical fields, Phys. Letters A 100, 423 (1984)

Ottinger H.C., Beyond Equilibrium Thermodynamics, Wiley (2005)

Ottinger H.C. and Grmela M., Dynamics and thermodynamics of complex fluids: II Illustrations of a general formalism, Phys. Rev. E 55, 6633-6655 (1997)

Salmon J.-B., Colin A., Manneville S., Velocity profiles in shear-banding wormlike micelles, Phys. Rev. Lett. 90, 228303 (2003)

Schmitt V., Lequeux F., Pousse A., Roux D., Flow behavior and shear induced transition near an isotropic/nematic transition in equilibrium polymers Langmuir 10 955-961 (1994)

Vazquez P.A., McKinley G.H., Cook L.P., A network scission model for wormlike micellar solutions I. Model formulation and viscometric flow predictions, J. Non-Newtonian Fluid Mech. 144, 122-139 (2007)

Zhou L., Vazquez P.A., Cook L.P., Modeling the inhomogeneous response and formation of shear bands in steady and transient flows of entangled liquids, J, Rheol. 52, 591-623 (2008) 